\title{
Reorganization of learning-associated prefrontal synaptic plasticity between the recall of recent and remote fear extinction memory
}

\author{
Sandrine Hugues and Rene Garcia ${ }^{1}$ \\ Laboratoire de Neurobiologie \& Psychopathologie, JE2441, Université de Nice-Sophia Antipolis, Nice, 06108, France
}

\begin{abstract}
We have previously shown that fear extinction is accompanied by an increase of synaptic efficacy in inputs from the ventral hippocampus (vHPC) and mediodorsal thalamus (MD) to the medial prefrontal cortex (mPFC) and that disrupting these changes to mPFC synaptic transmission compromises extinction processes. The aim of this study was to examine whether these extinction-related changes undergo further plasticity as the memory of extinction becomes more remote. Changes in synaptic efficacy in both vHPC-mPFC and MD-mPFC inputs were consequently analyzed when the memory was either $1 \mathrm{~d}$ or $7 \mathrm{~d}$ old. Increases of synaptic efficacy in the vHPC-mPFC pathway were observed when the memory was $1 \mathrm{~d}$ old, but not $7 \mathrm{~d}$ after initial extinction. In contrast, potentiation of synaptic efficacy in the MD-mPFC pathway increased over time. In rats that received low-frequency vHPC stimulation immediately after extinction, both vHPC-mPFC and MD-mPFC inputs failed to develop potentiation, and the recall of extinction (both recent and remote memories) was impaired. These findings suggest that post-extinction potentiation in vHPC-mPFC inputs may be necessary for both the recall of recent memory and post-extinction potentiation in the MD-mPFC inputs. This late potentiation process may be required for the recall of remote extinction memory.
\end{abstract}

Synaptic plasticity in the form of long-term potentiation (LTP), first discovered in the hippocampus (HPC) (Bliss and Lomo 1973), can take place in the medial prefrontal cortex (mPFC) following tetanic stimulation of the HPC (Laroche et al. 2000), or the mediodorsal thalamus (MD) (Herry and Garcia 2002), or the basolateral amygdala (BLA) (Maroun and Richter-Levin 2003). LTP-like changes, which are believed to contribute to various learning and memory processes (e.g., Garcia et al. 1993; Rogan et al. 1997), have also been observed in the mPFC in relation with extinction learning. Indeed, both MD-mPFC (Herry and Garcia 2002) and HPC-mPFC (Farinelli et al. 2006; Hugues et al. 2006) pathways have been reported to display fear extinction learningrelated LTP.

Although recent findings have indicated that lesions of the mPFC do not always impair extinction learning and memory (Farinelli et al. 2006; Garcia et al. 2006), as previously reported (Morgan et al. 1993; Quirk et al. 2000), analyses of extinctionrelated changes in synaptic efficacy in the mPFC remain of particular interest for at least two main reasons. Firstly, studies using rodents have repeatedly shown that the levels of changes in prefrontal neuronal activity strongly correlate with the degree of expression of extinction memory (Herry and Garcia 2002; Milad and Quirk 2002; Farinelli et al. 2006; Hugues et al. 2006). Indeed, these investigators found that the lack of such changes or impairment of their development, with low-frequency stimulation (LFS) of mPFC inputs or drug infusions into the mPFC, is associated with full recovery of fear responses, indicating that LTP-like changes in the mPFC following extinction training are a good index of maintenance of extinction (Garcia 2002; Quirk et al. 2006). Second, HPC-cortical circuits have been reported to undergo a major reorganization between recent and remote stages of contextual fear memory (Frankland et al. 2004) and spatial memory (Bontempi et al. 1999; Maviel et al. 2004), which are

'Corresponding author.

E-mail rgarcia@unice.fr; fax 33-492-07-61-62.

Article is online at http://www.learnmem.org/cgi/doi/10.1101//m.625407. both hippocampal-dependent tasks. Given that fear extinction is also a hippocampal-dependent process (Vianna et al. 2001, 2003, 2004; Cannich et al. 2004; Corcoran et al. 2005; Farinelli et al. 2006), we hypothesized that extinction-related changes in mPFC inputs may also undergo plasticity as a function of extinction maturation.

In order to investigate this possibility, we examined changes in HPC-mPFC and MD-mPFC synaptic efficacy 1 and $7 \mathrm{~d}$ after extinction training. We then analyzed whether HPC LFS would interfere with both extinction-related synaptic plasticity in the mPFC and the recall of extinction memory 1 and $7 \mathrm{~d}$ after training.

\section{Results}

\section{Histology}

Verification of electrode placements revealed that all rats were well implanted with stimulating electrodes in either the MD or the vHPC (Fig. 1A) and recording electrodes in the mPFC (Fig. 1B).

\section{Hippocampal inputs to the mPFC}

Field potentials recorded in the mPFC following stimulation of the HPC (Fig. 2) were similar to responses that have been largely described elsewhere (Laroche et al. 2000; Izaki et al. 2003; Hugues et al. 2006; Okulski et al. 2007). One rat was excluded for instable responses over days. All other rats $(n=9)$ exhibited stable field potential responses across the $2 \mathrm{~d}$ of baseline recordings. Responses remained stable following fear conditioning (Fig. 2, PreExt recordings). Following extinction training, rats that did not receive vHPC LFS (NLFS group; $n=5$ ) exhibited potentiation of synaptic efficacy that was observed $24 \mathrm{~h}$, but not $7 \mathrm{~d}$, after training (Fig. 2, 1d-post and 7d-post, respectively). In contrast, rats that received vHPC LFS (LFS group; $n=4$ ) showed decreases in field potential amplitudes. A two-way ANOVA performed on these data (two groups, five recording periods) indicated a main 


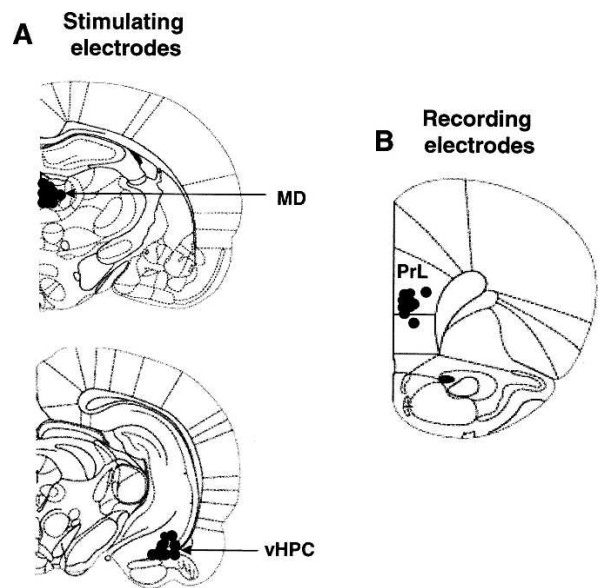

Figure 1. Diagrams showing electrode placements (filled circles) in the mediodorsal thalamus (MD) and ventral hippocampus (VHPC) for stimulation $(A)$, and mostly in the ventral part of the prelimbic area (PrL) of the medial prefrontal cortex for field potential recordings $(B)$.

effect of group $\left(F_{(1,7)}=19.6 ; P<0.01\right)$. There was also a significant interaction between group and recording period $\left(F_{(4,28)}=4.3 ; P<0.01\right)$. Post-hoc Scheffé tests revealed that the NLFS rats differed from the LFS group during the first postextinction delay $(P<0.05)$, the two groups being similar during the second post-extinction delay. In addition, changes in vHPCmPFC synaptic efficacy (potentiation) in NLFS rats were significant only during the first post-extinction delay.

\section{Thalamic inputs to the mPFC}

Field potentials recorded in the mPFC following stimulation of the MD (Fig. 3) were similar to responses previously described by our group (Herry et al. 1999; Herry and Garcia 2002, 2003). One rat was also excluded here for instability of responses over days. The nine other rats exhibited stable field potential responses across the $2 \mathrm{~d}$ of baseline recordings. Fear conditioning reduced amplitude of these responses. However, 1 and $7 \mathrm{~d}$ after extinction training, rats that did not received vHPC LFS (NLFS group; $n=5$ ) showed a slight and a large potentiation, respectively (Fig. 3, 1d-post and 7d-post). Rats that received vHPC LFS (LFS group; $n=4$ ) failed to display these changes. In contrast, depression of field potential amplitude was observed during the second postextinction delay (Fig. 3, 7d-post). A two-way ANOVA performed on these data (two groups, five recording periods) revealed a main effect of group $\left(F_{(1,7)}=48.3 ; P<0.001\right)$ and recording pe$\operatorname{riod}\left(F_{(4,28)}=3.8 ; P<0.05\right)$ and a group $\times$ recording period in- teraction $\left(F_{(4,28)}=16.1 ; P<0.0001\right)$. Post-hoc Scheffé tests indicated that the two groups (NLFS and LFS) differed from each other during both post-extinction delays (1d-post: $P<0.05$ and 7d-post: $P<0.01)$. Post-conditioning depression was significant in both groups (both $P<0.05$ ). Post-extinction analyses performed on data from the NLFS group indicated that potentiation was significant from the first post-extinction delay (1d-post: $P<0.01$ and 7d-post: $P<0.001)$. However, in the LFS group, the depression was significant only during the second postextinction delay $(P<0.05)$.

\section{Extinction and recovery of conditioned freezing behavior}

Before extinction (Fig. 4, pre-Ext) and each retention test (Fig. 4, pre-RT1 and pre-RT2), all rats exhibited low levels of freezing behavior. However, during early extinction (EE; five CS-alone presentations), both groups of rats (NLFS; $n=10$; LFS, $n=8$ ) displayed high levels of freezing to the tone CS (Fig. 4), indicating acquisition of auditory fear conditioning. Their freezing levels progressively decreased and reached low values during late extinction (EE; five CS-alone presentations). During each retention test, levels of freezing stayed at low values in rats that did not receive vHPC LFS (NLFS group). However, rats that received vHPC LFS (LFS group) displayed high levels of freezing behavior at each retention test, thus revealing difficulties to recall extinction (both recent and remote memories) in this group (Fig. 4, RT1 and RT2). Direct between-group analyses revealed that the two groups differed from each other only during the retention tests (1d-post: $P<0.0001$ and 7d-post: $P<0.01$ ).

\section{Discussion}

We found here that extinction of conditioned freezing to a tone $\mathrm{CS}$ in rats produced increases of synaptic efficacy in two different inputs to the mPFC, albeit with different time-courses. Potentiation of vHPC-mPFC synaptic efficacy was high $1 \mathrm{~d}$ after extinction training but was no longer present $7 \mathrm{~d}$ after training. However, mPFC potentiation of synaptic efficacy in MD afferents was present at both delays ( 1 and $7 \mathrm{~d}$ after training) but was greater at 7-d delay. In rats that received immediate post-training HPC LFS, we found not only suppression of extinction-associated changes in HPC-mPFC pathway but also inhibition of development of potentiation of synaptic efficacy in the MD-mPFC and also recovery of conditioned freezing behavior during each retention test.

Potentiation of neuronal activity in the mPFC following the suppression of conditioned fear responses (extinction training or treatment with antidepressants) has been widely reported in
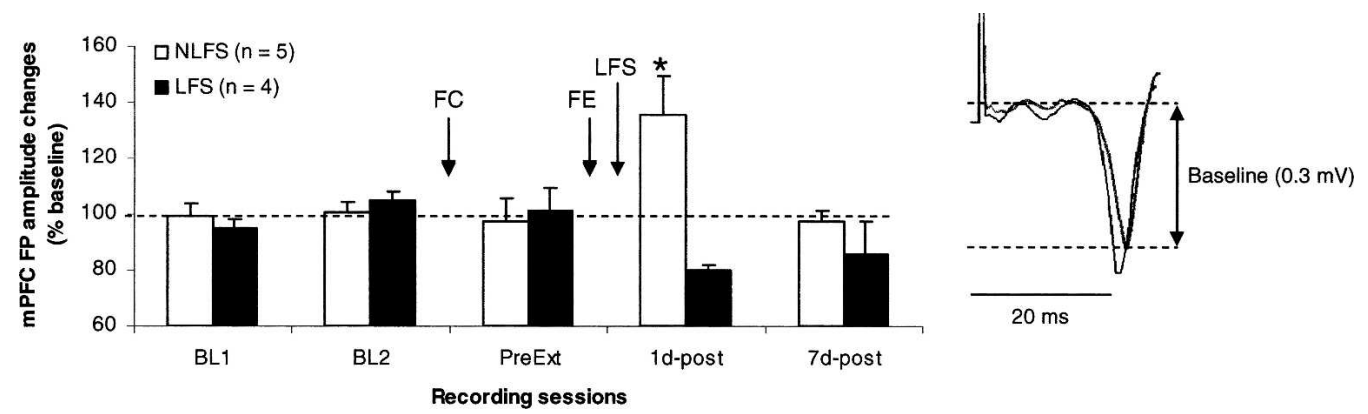

Figure 2. Changes (mean \pm SEM percentage relative to baseline) in the amplitude of hippocampal-prefrontal field potential (mPFC FP) during baseline (BL1 and BL2), pre-extinction training (PreExt), and at 1 and $7 \mathrm{~d}$ post-extinction delays (1d-post and 7d-post) in rats that received or did not receive hippocampal low-frequency stimulation (NLFS and LFS groups). (Left) Representative prefrontal field potentials recorded during baseline establishment and during $1 \mathrm{~d}$ after training. Changes in field potential amplitude were measured between the two dotted lines. FC indicates fear conditioning; FE, fear extinction. ${ }^{*} P<0.05$ (NLFS vs. LFS). 


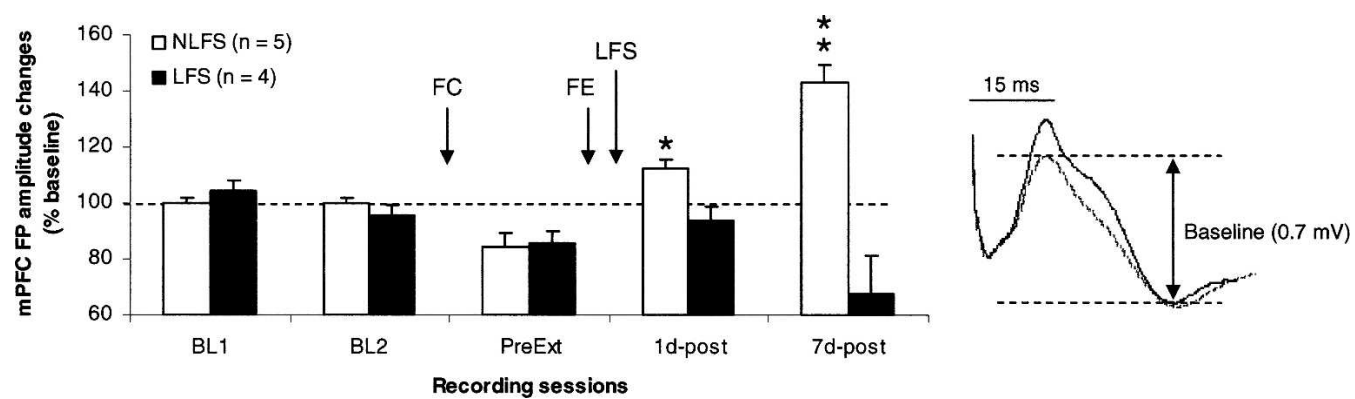

Figure 3. Changes (mean \pm SEM percentage relative to baseline) in the amplitude of mediodorsal thalamic-prefrontal field potential (mPFC FP) during baseline (BL1 and BL2), pre-extinction training (PreExt), and at 1 and $7 \mathrm{~d}$ post-extinction delays (1d-post and 7d-post) in rats that received or did not receive hippocampal low-frequency stimulation (NLFS and LFS groups). (Left) Representative prefrontal field potentials recorded during baseline establishment and during $1 \mathrm{~d}$ after training. Changes in field potential amplitude were measured between the two dotted lines. FC, fear conditioning; $\mathrm{FE}$, fear extinction. ${ }^{*} P<0.05 ;{ }^{* *} P<0.01$ (NLFS vs. LFS).

both rodents (Herry and Garcia 2002; Milad and Quirk 2002; Barrett et al. 2003; Herry and Mons 2004; Farinelli et al. 2006; Hugues et al. 2006) and humans (Fernandez et al. 2001; Seedat et al. 2004). Extinction-related potentiation in the MD-mPFC pathway was first reported in mice (Herry et al. 1999; Herry and Garcia 2002). Our present data confirm these findings in rats and show that extinction-related MD-mPFC potentiation develops progressively over a period of at least the week that followed training. Our present data also confirm that synaptic plasticity develop in the HPC-mPFC pathway in relation to fear extinction. However, in the two previous studies, these changes were observed within the $2 \mathrm{~h}$ (Hugues et al. 2006) and up to $3 \mathrm{~d}$ (Farinelli et al. 2006) after extinction training. In the present study, we found that these increases in vHPC-mPFC synaptic efficacy were no longer observed when the retention test was performed with a longer delay ( $7 \mathrm{~d}$ after training), at a time where MD-mPFC potentiation was higher, indicating major reorganization of levels of synaptic efficacy in mPFC inputs within the post-training time-period of $7 \mathrm{~d}$. Together, these observations indicate that extinction training first induced potentiation changes in the HPC-mPFC pathway, which declined with the passage of time, and was progressively replaced by potentiation of synaptic efficacy in another mPFC input. This shifting of potentiation changes from the HPC inputs to other inputs to the MPFC suggests possible transfer of extinction information from certain cortical inputs to others as the memory becomes more remote. A similar time-dependent reorganization of the neuronal networks mediating the dialog between the HPC and neocortex has been reported for HPC-dependent memory tasks such as spatial learn- ing and contextual fear conditioning. For spatial learning, it has been found that recall of recently acquired spatial information is associated with a selective activation of the HPC, whereas recall of remote spatial memory is associated with activation of the mPFC and anterior cingulate cortex (Bontempi et al. 1999; Maviel et al. 2004). Similarly, it has been observed that electrolytic lesions of the HPC produce a temporally graded retrograde amnesia for contextual fear memories (Kim and Fanselow 1992), while inactivation of the anterior cingulate cortex impairs remote contextual fear memory without affecting recent fear memory in the conditioning context (Frankland et al. 2004). As is the case for both spatial memory and contextual fear memory, fear extinction is also dependent on HPC functioning (Vianna et al. 2001, 2003, 2004; Corcoran et al. 2005).

We also found that suppression of early extinction-related HPC-mPFC potentiation, by means of HPC LFS, affected the development of delayed extinction-related potentiation in MDmPFC inputs. The HPC is reciprocally connected to MD (Su and Bentivoglio 1990). HPC LFS could have thus induced LTD in synaptic efficacy in the MD that, in turn, produced hypofunctionality of this structure in terms of inducing extinction-related synaptic changes in its cortical outputs. It may be possible to demonstrate this eventuality in animals additionally implanted with recording electrodes in the MD. Alternatively, as the two pathways studied here converge in the mPFC (Groenewegen et al. 1997), installation of LTD in the HPC-mPFC synapses may have produced associative LTD in other MPFC inputs that later compromised development of extinction-related LTP in these inputs. It may be possible to address this issue experimentally in

\section{Extinction training}

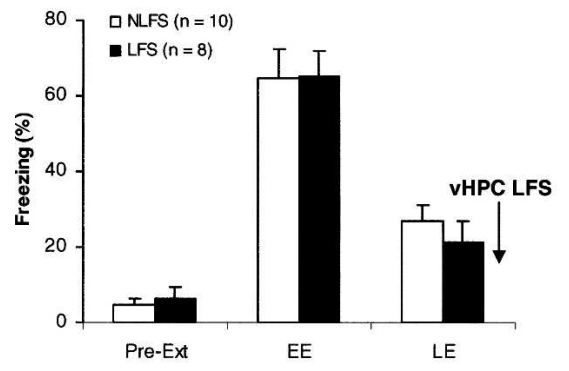

Retention tests (RT)

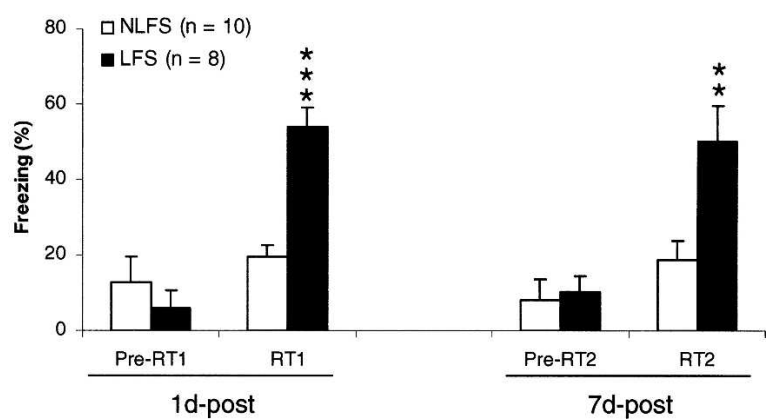

Figure 4. (Left, extinction training). Freezing behavior (mean \pm SEM) before the first CS presentation (pre-Ext), and during the first (early extinction, $\mathrm{EE}$ ) and last (late extinction, LE) five CS presentations of extinction training (15 tone-alone presentations). (Right, retention tests) Freezing before and during each retention test ( $1 \mathrm{~d}$-post and 7d-post; five tone-alone presentations/test) in rats that received or did not receive hippocampal low-frequency stimulation (NLFS and LFS groups). ${ }^{* *} P=0.01 ;{ }^{* * *} P<0.0001$ (NLFS vs. LFS). 
animals if it can be demonstrated that animals that do not display LTD in MD-mPFC pathway following MD LFS (Herry et al. 1999) only develop LTD when MD LFS is associated with HPC LFS.

Because we used a within-subjects design (i.e., the same rats were tested at $1 \mathrm{~d}$ and $7 \mathrm{~d}$ ), one can consider that any differences at $7 \mathrm{~d}$ were somewhat confounded by potential extinction occurring in the first retention test. However, animals completely extinguished their conditioned fear during initial extinction and did not show any improvement in extinction during the first retention test. In addition, animals that received HPC LFS completely recover their conditioned freezing during the second test despite the first test. We have also reported that animals tested directly $7 \mathrm{~d}$ after extinction training have both potentiation of MD-mPFC synaptic efficacy and same levels of freezing as during their late extinction (Herry and Garcia, 2002). In other words, it is likely that prior testing $(1 \mathrm{~d})$ in the present study did not affect responses to subsequent testing $(7 \mathrm{~d})$.

In conclusion, our findings provide evidence for the existence of differential changes in levels of synaptic efficacy in mPFC inputs between recall of a 1-d-old extinction memory and recall of a 7-d-old extinction memory. As a consequence, this reorganization, which depends on hippocampal functioning, would allow long-term maintenance of potentiation of neural activity in the mPFC. It has been suggested that increases of neural activity in the infralimbic area of the MPFC contribute to the activation of GABAergic intercalated cells (ITCs) within the amygdala. These ITCs, in turn, inhibit outputs of the central nucleus of the amygdala, thereby canceling fear expression during the recall of extinction (Quirk et al. 2006). However, in the present study, mPFC field potentials were recorded in the ventral part of the prelimbic area, where they were found higher in amplitude. Together with other findings (Sierra-Mercado et al. 2006; VidalGonzalez et al. 2006; Corcoran and Quirk 2007), it is likely that these field potentials reflected activity in the infralimbic area of the MPFC, although when the electrodes were lowered in the infralimbic region, the responses did not significantly increase in amplitude.

\section{Materials and Methods}

\section{Subjects and surgery}

Twenty male Wistar rats (Dépré, Saint Doulchard, France), weighing $280 \mathrm{~g}$ were housed in individual cages in room at $22^{\circ} \mathrm{C}$ with 12-h light/dark cycle and allowed food and water ad libitum. The experiments were designed in accordance to the European Community Guidelines on the care and use of laboratory animals (86/609/EEC). Five to seven days after arrival, each rat was anesthetized with sodium pentobarbital $(65 \mathrm{mg} / \mathrm{kg}, \mathrm{i} . \mathrm{p}$.) and placed in a stereotaxic frame. They were ipsilaterally implanted (right side) with a pair of twisted silver electrodes $(90-\mu \mathrm{m}$ diameter; insulated except at section) for field potential recording. The recording electrodes targeted the $\mathrm{mPFC}(3.2 \mathrm{~mm}$ anterior to bregma, $0.6 \mathrm{~mm}$ lateral to midline, and $3.2 \mathrm{~mm}$ from dura), and the stimulating electrodes targeted the CA1/ subiculum region of the ventral HPC (vHPC: $5.8-6.3 \mathrm{~mm}$ posterior to bregma, 5.3-5.6 $\mathrm{mm}$ lateral to midline, and $4.5-5.5 \mathrm{~mm}$ from dura). Half of these rats were also implanted with a third pair of electrodes in the MD $(2.5-2.8 \mathrm{~mm}$ posterior to bregma, $0.7 \mathrm{~mm}$ lateral to midline, and $4.0-4.5 \mathrm{~mm}$ from dura). One of three screws on the skull served as the recording ground. In addition to electrode placement in the brain, two stimulating electrodes (silver wires, 110- $\mu \mathrm{m}$ diameter, insulated except at the tip: 0.4-0.6 $\mathrm{mm}$ exposed) were inserted in the right eyelid for shock administration during fear conditioning. The entire miniature system was fixed in place onto the skull with dental cement.

\section{Stimulating and recording procedures}

After 4-7 d of recovery from surgery, rats were habituated, over a 2 -d period, to being transported (from the animal house to the experimental room) and to being connected to electrophysiological cables (relayed at the top of the experimental box by a multi-channel rotating connector, allowing free movement). Electrophysiological studies started after this period. Field potentials evoked in the mPFC by single-pulse stimulation (0.1-msec rectangular monophasic pulses) applied to the vHPC or MD were sent to an amplifier (gain $1000 \times$; bandpass $0.001-1 \mathrm{kHz}$ ) and recorded (Spike2 software; Cambridge Electronic Design) for offline analysis. Before the first baseline recording session, responses were measured as a function of stimulus strength (input-output curves: $100-800 \mu \mathrm{A}$ ). An intensity corresponding to $60 \%-70 \%$ of the saturation level was chosen for the test stimulus, which was applied every 5 sec during each recording session.

\section{Experimental protocol}

Baseline recording sessions (20 field potentials/session/day) took place in a box (box A, with four vertical sides, each $30 \mathrm{~cm}$ wide), which was washed with a solution containing a mixture of coconut and vanilla scent before introducing each animal. Thirty minutes following the second baseline recording session, each rat was then placed in another box (box B, with six vertical sides, one $30 \mathrm{~cm}$, three $25 \mathrm{~cm}$, and two $10 \mathrm{~cm}$ wide), which was washed with a solution containing a mixture of ethanol (50\%) and lemon scent. Boxes A and B were placed inside a soundattenuating and temperature-regulated chamber (Imetronic). Two minutes after being placed in box B, all rats implanted for electrophysiology (FC group) were subjected to five tone (30-sec/ 2.5-kHz/75-dB)-shock (a train of eight pulses/5 Hz/3.5 mA/1.4 sec) pairings (intertrial interval: $30-120 \mathrm{sec}$ ). After the last tone presentation, animals were divided into two groups: NLFS $(n=10)$ and LFS $(n=10)$. Rats of the LFS group were immediately submitted to VHPC LFS that consisted of a train of pulses at $2 \mathrm{~Hz}$ for $25 \mathrm{~min}$. The NLFS rats were also left in box B for $25 \mathrm{~min}$ but did not receive LFS. The following day, each rat was placed in box A, where 15 tone-alone presentations were delivered (intertrial interval: $30-120 \mathrm{sec}$ ). Retention tests took also place in box A, 1 and $7 \mathrm{~d}$ following extinction training; rats were exposed to five tone-alone presentations at each post-extinction test.

Field potentials (20/session) were recorded in box A before each first presentation of the tone during extinction training and each retention test. The behavior of each rat was continuously monitored and recorded via a camera-VCR-monitor system. Conditioned fear was assessed by measuring freezing behavior, defined as the absence of all movement except for respiratoryrelated movements (Blanchard and Blanchard 1969), during the $32 \mathrm{sec}$ preceding the first CS-alone presentation (i.e., before extinction training and each retention test). Freezing was scored using a time-sampling procedure.

\section{Histology and data analyses}

On completion of the experiments, rats were placed under deep anesthesia, and the tips of electrode placements in the mPFC, MD or HPC were marked by passing 0.3- to $0.5-\mathrm{mA}$ current for 20 sec. The placement of the electrodes was then verified by standard histological methods. ANOVA.

All data were expressed as means and SEM and analyzed by

\section{Acknowledgments}

We thank Dr. T. Durkin for critical reading of the manuscript. This study was supported by the University of Nice-Sophia Antipolis and a grant from the Délégation Générale pour l'Armement to S.H.

\section{References}

Barrett, D., Shumake, J., Jones, D., and Gonzalez-Lima, F. 2003. Metabolic mapping of mouse brain activity after extinction of a conditioned emotional response. J. Neurosci. 23: 5740-5749. 
Blanchard, R.J. and Blanchard, C. 1969. Crouching as an index of fear. J. Comp. Physiol. Psychol. 6: 370-375.

Bliss, T.V. and Lomo, T. 1973. Long-lasting potentiation of synaptic transmission in the dentate area of the anaesthetized rabbit following stimulation of the perforant path. J. Physiol. 232: 331-356.

Bontempi, B., Laurent-Demir, C., Destrade, C., and Jaffard, R. 1999. Time-dependent reorganization of brain circuitry underlying long-term memory storage. Nature 400: 671-675.

Cannich, A., Wotjak, C.T., Kamprath, K., Hermann, H., Lutz, B., and Marsicano, G. 2004. CB1 cannabinoid receptors modulate kinase and phosphatase activity during extinction of conditioned fear in mice. Learn. Mem. 11: 625-632.

Corcoran, K.A. and Quirk, G.J. 2007. Recalling safety: Cooperative functions of the ventromedial prefrontal cortex and the hippocampus in extinction. CNS Spectr. 12: 200-206.

Corcoran, K.A., Desmond, T.J., Frey, K.A., and Maren, S. 2005. Hippocampal inactivation disrupts the acquisition and contextual encoding of fear extinction. J. Neurosci. 25: 8978-8987.

Farinelli, M., Deschaux, O., Hugues, S., Thevenet, A., and Garcia, R. 2006. Hippocampal train stimulation modulates recall of fear extinction independently of prefrontal cortex synaptic plasticity and lesions. Learn. Mem. 13: 329-334.

Fernandez, M., Pissiota, A., Frans, O., von Knorring, L., Fischer, H., and Fredrikson, M. 2001. Brain function in a patient with torture related post-traumatic stress disorder before and after fluoxetine treatment: A positron emission tomography provocation study. Neurosci. Lett. 297: 101-104.

Frankland, P.W., Bontempi, B., Talton, L.E., Kaczmarek, L., and Silva, A.J. 2004. The involvement of the anterior cingulate cortex in remote contextual fear memory. Science 304: 881-883.

Garcia, R. 2002. Postextinction of conditioned fear: Between two CS-related memories. Learn. Mem. 9: 361-363.

Garcia, R., Vouimba, R.M., and Jaffard, R. 1993. Spatial discrimination learning induces LTP-like changes in the lateral septum of mice. Neuroreport 5: 329-332.

Garcia, R., Chang, C.H., and Maren, S. 2006. Electrolytic lesions of the medial prefrontal cortex do not interfere with long-term memory of extinction of conditioned fear. Learn. Mem. 13: 14-17.

Groenewegen, H.J., Wright, C.I., and Uylings, H.B. 1997. The anatomical relationships of the prefrontal cortex with limbic structures and the basal ganglia. J. Psychopharmacol. 11: 99-106.

Herry, C. and Garcia, R. 2002. Prefrontal cortex long-term potentiation, but not long-term depression, is associated with the maintenance of extinction of learned fear in mice. J. Neurosci. 22: 577-583.

Herry, C. and Garcia, R. 2003. Behavioral and paired-pulse facilitation analyses of long-lasting depression at excitatory synapses in the medial prefrontal cortex in mice. Behav. Brain Res. 146: 89-96.

Herry, C. and Mons, N. 2004. Resistance to extinction is associated with impaired immediate early gene induction in medial prefrontal cortex and amygdala. Eur. J. Neurosci. 20: 781-790.

Herry, C., Vouimba, R.M., and Garcia, R. 1999. Plasticity in the mediodorsal thalamo-prefrontal cortical transmission in behaving mice. J. Neurophysiol. 82: 2827-2832.

Hugues, S., Chessel, A., Léna, I., Marsault, R., and Garcia, R. 2006. Prefrontal infusion of PD098059 immediately after fear extinction training blocks extinction-associated prefrontal synaptic plasticity and decreases prefrontal ERK2 phosphorylation. Synapse 60: $280-287$.

Izaki, Y., Takita, M., Nomura, M., and Akema, T. 2003. Differences between paired-pulse facilitation and long-term potentiation in the dorsal and ventral hippocampal CA1-prefrontal pathways of rats.
Brain Res. 992: 142-145.

Kim, J.J. and Fanselow, M.S. 1992. Modality-specific retrograde amnesia of fear. Science 256: 675-677.

Laroche, S., Davis, S., and Jay, T.M. 2000. Plasticity at hippocampal to prefrontal cortex synapses: Dual roles in working memory and consolidation. Hippocampus 10: 438-446.

Maroun, M. and Richter-Levin, G. 2003. Exposure to acute stress blocks the induction of long-term potentiation of the amygdala-prefrontal cortex pathway in vivo. J. Neurosci. 23: 4406-4409.

Maviel, T., Durkin, T.P., Menzaghi, F., and Bontempi, B. 2004. Sites of neocortical reorganization critical for remote spatial memory. Science 305: 96-99.

Milad, M.R. and Quirk, G.J. 2002. Neurons in medial prefrontal cortex signal memory for fear extinction. Nature 420: 70-74.

Morgan, M.A., Romanski, L.M., and LeDoux, J.E. 1993. Extinction of emotional learning: contribution of medial prefrontal cortex. Neurosci. Lett. 163: 109-113.

Okulski, P., Jay, T.M., Jaworski, J., Duniec, K., Dzwonek, J., Konopacki, F.A., Wilczynski, G.M., Sanchez-Capelo, A., Mallet, J., and Kaczmarek, L. 2007. TIMP-1 abolishes MMP-9-dependent long-lasting long-term potentiation in the prefrontal cortex. Biol. Psychiatry doi:10.1016/j.biopsych.2006.09.012.

Quirk, G.J., Russo, G.K., Barron, J.L., and Lebron, K. 2000. The role of ventromedial prefrontal cortex in the recovery of extinguished fear. J. Neurosci. 20: 6225-6231.

Quirk, G., Garcia, R., and Gonzalez-Lima, F. 2006. Prefrontal mechanisms in extinction of conditioned fear. Biol. Psychiatry 60: $337-343$.

Rogan, M.T., Staubli, U.V., and LeDoux, J.E. 1997. Fear conditioning induces associative long-term potentiation in the amygdala. Nature 390: 604-607.

Seedat, S., Warwick, J., van Heerden, B., Hugo, C., Zungu-Dirwayi, N., Van Kradenburg, J., and Stein, D.J. 2004. Single photon emission computed tomography in posttraumatic stress disorder before and after treatment with a selective serotonin reuptake inhibitor. $J$. Affect. Disord. 80: 45-53.

Sierra-Mercado Jr., D., Corcoran, K.A., Lebron-Milad, K., and Quirk, G.J. 2006. Inactivation of the ventromedial prefrontal cortex reduces expression of conditioned fear and impairs subsequent recall of extinction. Eur. J. Neurosci. 24: 1751-1758.

$\mathrm{Su}$, H.S. and Bentivoglio, M. 1990. Thalamic midline cell populations projecting to the nucleus accumbens, amygdala, and hippocampus in the rat. J. Comp. Neurol. 297: 582-593.

Vianna, M.R., Szapiro, G., McGaugh, J.L., Medina, J.H., and Izquierdo, I. 2001. Retrieval of memory for fear-motivated training initiates extinction requiring protein synthesis in the rat hippocampus. Proc. Natl. Acad. Sci. 98: 12251-12254.

Vianna, M.R., Igaz, L.M., Coitinho, A.S., Medina, J.H., and Izquierdo, I. 2003. Memory extinction requires gene expression in rat hippocampus. Neurobiol. Learn. Mem. 79: 199-203.

Vianna, M.R., Coitinho, A.S., and Izquierdo, I. 2004. Role of the hippocampus and amygdala in the extinction of fear-motivated learning. Curr. Neurovasc. Res. 1: 55-60.

Vidal-Gonzalez, I., Vidal-Gonzalez, B., Rauch, S.L., and Quirk, G.J. 2006. Microstimulation reveals opposing influences of prelimbic and infralimbic cortex on the expression of conditioned fear. Learn. Mem. 13: 728-733.

Received May 10, 2007; accepted in revised form June 19, 2007. 


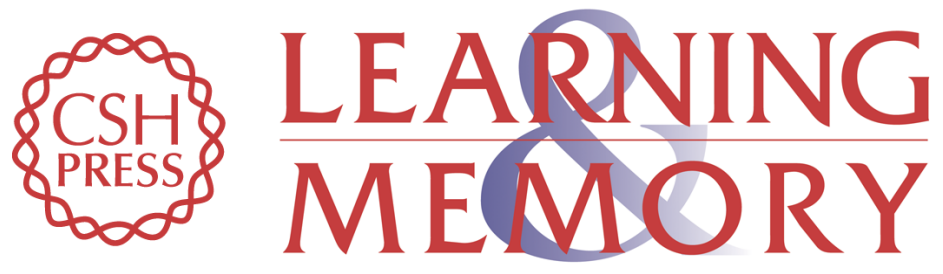

\section{Reorganization of learning-associated prefrontal synaptic plasticity between the recall of recent and remote fear extinction memory}

Sandrine Hugues and Rene Garcia

Learn. Mem. 2007, 14:

Access the most recent version at doi:10.1101//m.625407

References This article cites 36 articles, 14 of which can be accessed free at: http://learnmem.cshlp.org/content/14/8/520.full.html\#ref-list-1

License

Email Alerting

Receive free email alerts when new articles cite this article - sign up in the box at the Service top right corner of the article or click here. 